Reprod. Nutr. Dévelop., 1987, 27 (1 B), 253-254.

\title{
Effets de l'introduction d'une partie du lait dans le rumen du veau préruminant sur le fonctionnement de la gouttière csophagienne et la composition du jus de rumen
}

\author{
I. NUNES DO PRADO, R. TOULLEC, Y. MANIS, P. GUILLOTEAU \\ Laboratoire du Jeune Ruminant, \\ I.N.R.A., 65, rue de Saint-Brieuc, 35042 Rennes Cedex.
}

Summary. Pouring $25 \%$ of the milk into the rumen of preruminant calves resulted in a large and long-lasting decrease of rumen juice $\mathrm{pH}$ and an increase of the organic acid contents. However, neither feed intake nor the efficiency of oesophageal groove closure was altered.

Chez le veau préruminant, le lait ingéré passe directement dans la caillette, grâce au réflexe de fermeture de la gouttière œsophagienne (Guilhermet, Mathieu et Toullec, 1975). Cependant, chez les jeunes veaux qui s'adaptent mal en atelier d'engraissement, une part importante du lait ingéré tombe dans le rumen (Breukink et al., 1985 ; Tadeu Dos Santos et al., 1986). Le but de ce travail était d'étudier les effets de l'introduction d'une partie du lait dans le rumen sur l'appétit, le fonctionnement de la gouttière osophagienne et la composition du jus de rumen.

Matériel et méthodes. Trois veaux femelles Holstein, élevés sur caillebotis, ont été munis d'une canule du rumen à l'âge de 26 ( 2 veaux) ou $42 \mathrm{j}$ ( 1 veau). Les animaux ont reçu un lait de remplacement riche en poudre de lait écrémé qui a été normalement distribué au seau, en 2 repas/j, apportant au total $17,2 \mathrm{~g}$ de matière sèche et $85,7 \mathrm{~g} \mathrm{~d}$ 'eau $/ \mathrm{kg}$ de poids vif (PV). Le traitement expérimental a consisté à n'offrir que $75 \%$ de la ration par voie buccale et à introduire le reste dans le rumen, par la canule, immédiatement après la buvée. II a commencé 3 semaines après l'opération et a duré $\mathbf{3}$ semaines. II a été précédé d'une période préexpérimentale d'une semaine et d'une période postexpérimentale de 2 semaines pendant lesquelles la totalité de la ration a été distribuée par voie buccale. En fait, ces 2 périodes ont fourni des résultats identicues et ont donc été regroupées en une seule période témoin de 3 semaines. L'efficacité de fermeture de la gouttière œsophagienne a été testée 1 fois par semaine, en utilisant du polyéthylène glycol 4000 (PEG) comme marqueur, selon les modalités décrites par Guilhermet, Mathieu et Toullec (1975). Chaque test a comporté $2 \mathrm{j}$ de mesures pendant lesquels du jus de rumen a été prélevé avant le repas et 1, 2, 3, 4, 6 et $8 \mathrm{~h}$ après. Le $\mathrm{pH}$ et la concentration de PEG ont été déterminés dans tous les échantilloris, les acides gras volatils (AGV), I'acide lactique et l'ammoniac dans des échantillons moyens par veau, par temps de prélèvement et par période.

Résultats et discussion. L'introduction d'une partie du lait dans le rumen n'a pas eu d'effet sur l'appétit. Elle n'a pas non plus modifié l'efficacité de fermeture de la gouttière œsophagienne puisque le pourcentage de lait ingéré par voie buccale tombé dans le rumen est resté très faible (moyennes \pm écarts-types des moyennes : 1,0 $\pm 0,1$ au lieu de 1,3 $\pm 0,2$ pendant la période témoin). En revanche, le volume du liquide ruminal, déterminé après le repas, a été plus élevé $(\mathrm{P}<0,01)$ pendant la période expérimentale $(17,8 \pm 4,1 \mathrm{ml} / \mathrm{kg} \mathrm{PV}$ au lieu de $5,7 \pm 2,0)$. Le pH du jus de rumen a toujours baissé après le repas, mais davan- 
tage et pendant plus longtemps lors de la période expérimentale (fig. 1). Cela a été dû à un accroissement beaucoup plus fort des teneurs en acides organiques, particulièrement en acides $D$ et L-lactiques, alors que la teneur en ammoniac est demeurée plus faible. L'acidité organique totale obtenue $2 \mathrm{~h}$ après le repas est restée 2,7 fois moins élevée que celle observée au maximum par Ehouinsou (1976) dans le jus de rumen de moutons ruminants recevant un régime contenant $35 \%$ de lactose. Malgré cela, le $\mathrm{pH}$ a été aussi bas que chez le mouton, ce qui a probablement été dû à la sécrétion salivaire plus faible et de moindre pouvoir tampon du veau préruminant. Cependant, l'acidité a continué à croître au-delà de $2 \mathrm{~h}$ puisque le $\mathrm{pH}$ a baissé jusqu'à $4 \mathrm{~h}$. Le lactose a constitué une part élevée mais décroissante de la matière sèche durant les premières heures postprandiales de la période expérimentale $(40,7 \pm 0,5, \quad 35,5 \pm 1,2, \quad 18,3 \pm 0,8,13,1 \pm 1,1$, $1,1 \pm 0,3$ et $0,5 \pm 0,1 \%$ respectivement à $1, \overline{2}, 3,4,6$ et $8 \bar{h}$ ), alors qu'il a toujours été absent pendant la période témoin. Par ailleurs, du jus de rumen s'échappait de la canule quand les veaux étaient couchés. Des récoltes, effectuées pendant 10 journées à la fin de la période témoin, ont montré que cela équivalait à environ $35 \%$ des quantités de liquide ingéré.
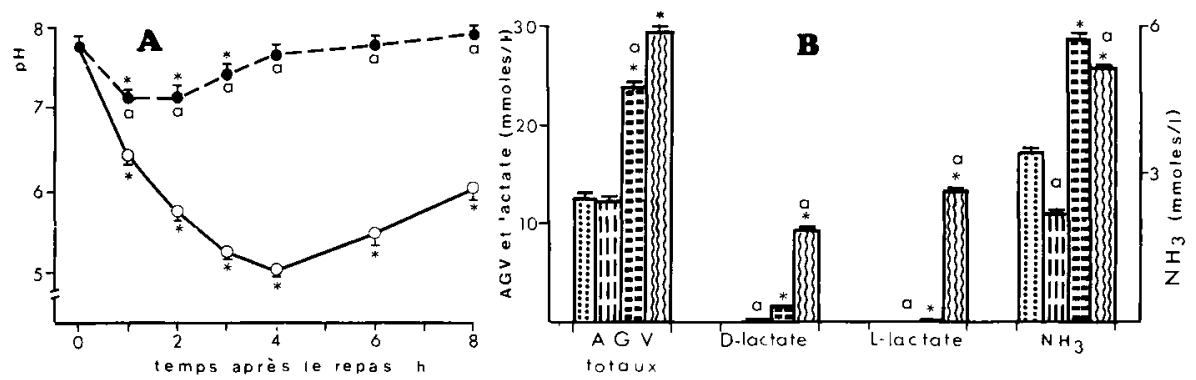

FIG. 1. - Evolution du $p H$ (A) et de la composition (B) du jus de rumen.

$\mathrm{A}$ : périodes témoin ( $)$ et expérimentale (O). B : période témoin à jeun (:: ) et $2 \mathrm{~h}(::)$ après le repas, période expérimentale à jeun ( III ) et $2 \mathrm{~h}$ ( $(\mathrm{l}$ ) après le repas. A et $\mathrm{B}$ : différence significative $(\mathrm{P}<0,05)$ entre périodes $(a)$ et par rapport à la valeur à jeun $\left({ }^{*}\right)$.

En conclusion, l'introduction d'une partie du lait dans le rumen a entraîné des fermentations importantes. Cependant, cela n'a pas altéré l'appétit et le fonctionnement de la gouttière œsophagienne, peut-être parce que la canule pouvait constituer un exutoire et que nos animaux n'avaient pas subi de stress, contrairement aux jeunes veaux arrivant en atelier d'engraissement.

Breukink H. J., Wensing T., Van Bruinessen-Kapsenberg E. G., de Visser N.A.P.C., WeerenKeverling Buisman A., 1985. In Ooms L.A.A., Degrysse A. D., Marsboom R., The ruminant stomach, 313-324. The Janssen Found., Antwerp.

Ehouinsou M. A., 1976. Thèse de Docteur-Ingénieur. Úniversité de Clermont-Ferrand. Série $D_{1}$. $104 \mathrm{pp}$.

Guilhermet R., Mathieu C. M., Toullec R., 1975. Ann. Zootech., 24, 69-79.

Tadeu Dos Santos G., Toullec R., Roger C., de la Grange H., Guilloteau P., 1986. Reprod. Nutr. Dévelop., 26, 1217. 